\title{
Contaminación producida por piscicultura intensiva en lagunas andinas de Junín, Perú
}

\author{
Pollution produced by intensive fish farming in Andean lagoons, Junín, \\ Peru
}

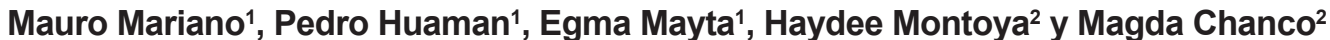

\begin{abstract}
1 Laboratorio de Fauna Dulceacuícola, Facultad de Ciencias Biológicas, Universidad Nacional Barón Mayor de San Marcos. Apartado postal 110058, Lima 11, Perú Email Mauro Mariano: mauroeg2002@yahoo.es 2 Museo de Historia Natural, Universidad Nacional Mayor de San Marcos.

Trabajo presentado a la XVIII Reunión Científica del Instituto de Investigaciones en Ciencias Biológicas Antonio Raimondi, "200 años del nacimiento de Charles Darwin y el 150 aniversario de la publicación de On the Origin of Species by Means of Natural Selection". Del 19 al 21 de agosto de 2009 .
\end{abstract}

Publicado impreso: 20/10/2010 Publicado online: $\quad$ 29/09/2010

\section{Resumen}

Se reportan los cambios producidos por el cultivo intensivo de la trucha Oncorhynchus mykiss en siete lagunas andinas. Las observaciones se realizaron en el año 1996, y entre el 2002 - 2007 y permitieron observar el proceso de deterioro de las lagunas, caracterizado por el incremento en las concentraciones de fosforo total y la disminución del oxigeno disuelto y la transparencia. La comunidad béntica fue evaluada en las siete lagunas en el 2007 , resultando el número de especies y los índices de diversidad bajos ( $H^{\prime}<1,26$; $<8$ spp.). La abundancia varió entre 7 y 35 ind. $/ 0,04 \mathrm{~m}^{2}$. La materia orgánica y carbonatos en fondo fueron altos $(30,22-42,45 \%)$.

Palabras clave: contaminación, lagunas andinas, cultivo de truchas, especies introducidas.

\section{Abstract}

We report the changes produced by intensive farming of rainbow trout Oncorhynchus mykiss in seven Andean lagoons. The observations were made in 1996 and between 2002-2007 and allowed to observe the deterioration of the lakes, characterized by the increase in total phosphorus concentrations and decreased dissolved oxygen and transparency. The benthic community was evaluated in seven lakes in 2007, resulting low number of species and diversity indices $\left(H^{\prime}<1,26 ;<8 \mathrm{spp}\right.$.). The abundance ranged from 7 to 35 ind. $/ 0,04 \mathrm{~m}^{2}$. Organic matter and carbonates were high in the bottom (from 30,22 to $42,45 \%$ ).

Keywords: contamination, Andean lagoon, trout culture, alien species.

\section{Introducción}

Las lagunas altoandinas son un rasgo fisiográfico muy importante de la región Junín, en ellas se desarrollan diferentes actividades económicas. Algunas de ellas se han visto afectadas como consecuencia de los cultivos intensivos de trucha arco iris (Oncorhynchus mykiss) que en ellas se realizan.

Las lagunas Tranca Grande, Cucancocha, Ayhuin, Pomacocha, Tipicocha, Habascocha y Huascacocha, localizados entre las vertientes orientales de la cuenca alta del Perene y occidentales de la cuenca del Mantaro, tuvieron un impacto humano moderado hasta hace unos años; sin embargo desde 1996 se inició el cultivo de trucha en jaulas flotantes, y engordadas con alimentos balanceados peletizados los cuales sumados a los desechos fecales constituyen un importante aporte de materia orgánica al ecosistema de las lagunas.

Cornell \& Whoriskey (1993) y Buschmann (2001) reportaron casos de contaminación de lagos donde se cultivaban salmones y truchas arco iris en sistemas de jaulas y que resultaron en la eutrofización del ecosistema y el fracaso de la empresa.

En el presente trabajo se describe el deterioro observado desde 1995 al 2007 en siete lagunas andinas donde se realiza cultivo intensivo de trucha

\section{Áreas de estudio}

Siete lagunas andinas fueron estudiadas desde 1996 hasta el 2007. Las lagunas se sitúan en el departamento de Junín, en los Andes Centrales del Perú, en la cuenca alta del Perene (Tranca Grande, Habascocha, Pomacocha, Tipicocha, Huascacocha) y del Mantaro (Cucancocha y Ayhuin). Alrededor de ellas se desarrolla una ganadería extensiva (ovino, vacuno y auquénido). Con escasa vegetación natural tanto en áreas alejadas de las lagunas como alrededor de éstas. (Fig. 1; Tabla 1)

Todas las lagunas están ubicadas entre 4310 y $4330 \mathrm{~m}$ de altitud; el clima presenta dos periodos: lluvioso (octubre-abril), en el que alcanzan el máximo nivel y periodo seco (mayo - setiembre) (SEDAPAL, 1999). La precipitación anual oscila entre 80 y $110 \mathrm{~mm}$ anuales, la temperatura varía desde los -2 hasta $16{ }^{\circ} \mathrm{C}$. La temperatura de las lagunas varía entre 10 y $12^{\circ} \mathrm{C}$, las profundidades son mayores de $10 \mathrm{~m}$, turbidez baja y buena

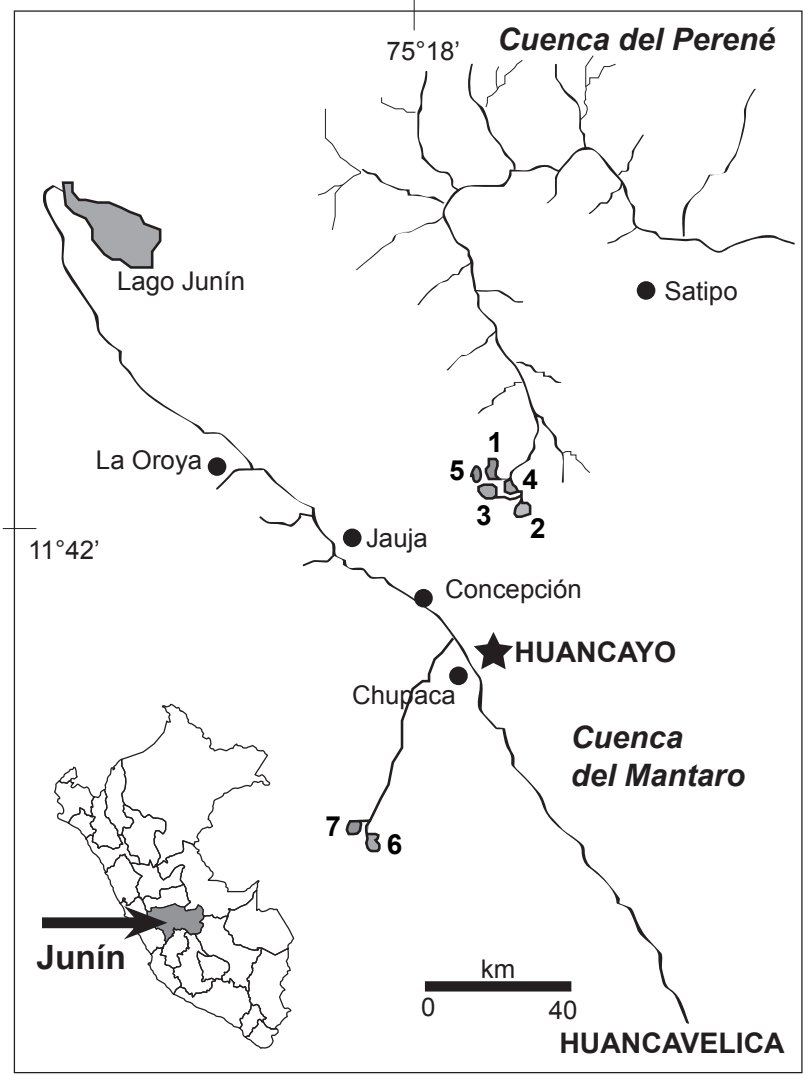

Figura 1. Ubicación de las cuencas del Perene y Mantaro mostrando las lagunas estudiadas: 1) Tranca Grande, 2) Habascocha,3) Pomacocha, 4) Tipicocha, 5) Huascacocha, 6) Cucancocha y 7) Ayhuin. 
Tabla 1. Principales características morfométricas de las lagunas altoandinas estudiadas, en Junín, Perú

\begin{tabular}{|c|c|c|c|c|c|c|}
\hline \multirow{2}{*}{$\begin{array}{l}\text { Lagunas } \\
\text { Tranca grande }\end{array}$} & \multicolumn{2}{|c|}{ Posición geográfica } & \multirow{2}{*}{$\begin{array}{c}\text { Altitud (m) } \\
4320\end{array}$} & \multirow{2}{*}{$\begin{array}{c}\begin{array}{c}\text { Área de la laguna } \\
\text { (ha) }\end{array} \\
164\end{array}$} & \multirow{2}{*}{$\begin{array}{c}\text { Profundidad } \\
\text { media (m) }\end{array}$} & \multirow{2}{*}{$\begin{array}{c}\text { Desarrollo de } \\
\text { línea de costa }\end{array}$} \\
\hline & $11^{\circ} 46^{\prime} 57 \mathrm{~S}$ & $75^{\circ} 14^{\prime} 42 \mathrm{~W}$ & & & & \\
\hline Habascocha & $11^{\circ} 47^{\prime} 26 \mathrm{~S}$ & $75^{\circ} 13^{\prime} 42 \mathrm{~W}$ & 4330 & 80 & 12 & 1,30 \\
\hline Pomacocha & $11^{\circ} 44^{\prime} 50 \mathrm{~S}$ & $75^{\circ} 13^{\prime} 40 \mathrm{~W}$ & 4310 & 120 & 9 & 1,40 \\
\hline Huascacocha & $11^{\circ} 43^{\prime} 45 S$ & $75^{\circ} 13^{\prime} 44 W$ & 4330 & 110 & 11 & 1,60 \\
\hline Tipicocha & $11^{\circ} 43^{\prime} 40 \mathrm{~S}$ & $75^{\circ} 15^{\prime} 08 W$ & 4320 & 90 & 10 & 1,45 \\
\hline Cucancocha & $12^{\circ} 08^{\prime} 19 S$ & $75^{\circ} 36^{\prime} 37 \mathrm{~W}$ & 4320 & 110 & 15 & 2,05 \\
\hline Ayhuin & $12^{\circ} 06^{\prime} 395$ & $75^{\circ} 38^{\prime} 01 \mathrm{~W}$ & 4320 & 105 & 15 & 1,78 \\
\hline
\end{tabular}

oxigenación, son lagunas polimicticas y su volumen y extensión variables. En todas las lagunas se observó la presencia de parches de macrofitas en la zona litoral y un sedimento arenoso. Se registraron peces nativos en varios muestreos; por ejemplo Orestias agassii estuvo presente en las lagunas de Tranca Grande, Cucancocha y Ayhuin.

El cultivo en jaulas fue iniciado en Tranca Grande en el año 1995, y en las otras seis lagunas en 1996. Todas las lagunas en estudio son administradas por particulares, excepto Tranca Grande que lo es por la Dirección Regional de Producción (DIREPRO). Las jaulas tienen volúmenes entre $56 \mathrm{~m}^{3}$ (para alevines y juveniles) y de $87,5 \mathrm{~m}^{3}$ (engorde), se ubican en la zona pelágica, con 6 a $20 \mathrm{~m}$ aproximadamente entre el fondo y la jaula. Están construidas con palos de eucaliptos o caña de Guayaquil, red-bolsa, mallas de nylon y cilindros de aluminio o plástico. El nivel de producción es de 10 a 20 Tm/año. La conversión alimenticia de 1,3:1; el tiempo de crianza en la laguna para ser comercializado de diez meses promedio.

Las condiciones de cultivo para la fase de producción de engorde fueron: densidades de siembra (alevinaje) de $126 \mathrm{~kg} /$ $\mathrm{m}^{3}$ (153 truchas $/ \mathrm{kg}$ de $8 \mathrm{~cm}$ ), de 10 a $12{ }^{\circ} \mathrm{C}$ de temperatura del agua, 40 a 80 ppm de dureza, con valores mínimos de $7 \mathrm{mg} / \mathrm{L} \mathrm{de}$ oxígeno disuelto y $\mathrm{pH}$ de 7 a 8 . Las tallas comerciales se lograron a los 10 meses de cultivo con $250 \mathrm{~g}$, observándose supervivencia de 90 a $95 \%$ y una tasa de conversión alimenticia de 1,4.

\section{Material y métodos}

Se realizaron colectas de agua para determinar fosforo total, oxigeno disuelto, transparencia, y de sedimento para determinar el tamańo de grano, en febrero, mayo, agosto y octubre; de los años 1996 al 2007; en una estación fija en la zona de mayor profundidad de las lagunas (aproximadamente a $50 \mathrm{~m}$ de las jaulas de cultivo).

Las mediciones del fósforo total se realizaron por el método del ácido ascórbico luego de una digestión con persulfato de potasio (APHA 1993). La concentración del oxígeno disuelto con el método de Winkler modificado por Carrit \& Carpenter (1966). La transparencia empleando el disco de Secchi. La materia orgánica y los carbonatos se determinaron mediante el método de Dean (1974). El tamańo de partícula del sedimento $(\mu \mathrm{m})$ se analizó de acuerdo a Krumbein \& Pettijohn (1938) calculadas con las ecuaciones obtenidas a partir de la velocidad de asentamiento de Stokes.

El macrobentos y materia orgánica total y carbonatos fueron determinados en las siete lagunas solamente el ańo 2007. Para la colecta se utilizó una draga Ekman $\left(0,04 \mathrm{~m}^{2}\right)$, con tres replicas por estación; cada replica fue tamizada con mallas $0,5 \mathrm{~mm}$ y conservados en formol al $10 \%$ en frascos para su posterior de- terminación taxonómica y análisis cualitativos y cuantitativos (Huamán et al. 2002). Una de las réplicas de sedimento fue utilizada para determinar el tamaño de grano, la materia orgánica y los carbonatos.

Los recuentos de organismos fueron realizados bajo microscopio estereoscopio en el laboratorio. Los valores de abundancia del macrobentos fueron expresados en individuos $/ 0,04 \mathrm{~m}^{2}$

Los índices de diversidad H' de Shannon, equitabilidad J' de Pielou, el índice de Margalef para la riqueza de especies y el índice de dominancia de Berger-Parker fueron calculados según Magurran (1988).

El análisis de agrupamiento se realizo a partir de la matriz de abundancia de macrobentos y utilizando la distancia Euclidiana y el algoritmo UPGMA.

\section{Resultados y discusión}

Las concentraciones del fosforo total se incrementaron desde 1996 al 2007; en el mismo periodo, el oxígeno disuelto y la transparencia disminuyeron en todas las lagunas. Los rangos de variación fueron los siguientes: fosforo total de $1-152 \mu \mathrm{g} / \mathrm{L}$, oxígeno disuelto en el fondo de $0,7-8 \mathrm{mg} / \mathrm{L}$ y la transparencia de $0,4-7,4 \mathrm{~m}$ (Tabla 2).

Durante el periodo de estudio, el tamaño de grano disminuyó de $960 \mu \mathrm{m}$, en 1996, a menor de $62 \mu \mathrm{m}$ (cieno) en el 2007 (Tabla 2). El cieno fue de coloración blanca y desprendía un fuerte olor a ácido sulfhídrico.

En las lagunas Habascocha, Pomacocha, Huascacocha y Tipicocha la materia orgánica total y los carbonatos en el sedimento se fueron 42,45 y $37,63 \%$ respectivamente. En las lagunas Tranca grande, Cucancocha y Ayhuin tuvieron porcentajes menores, la materia orgánica fue 30,22 y los carbonatos $26,20 \%$.

La macrofauna estuvo conformada por diez especies: Dugesia sp. (Turbellaria); Tubifex tubifex, Limnodrilus sp. (Oligochaeta); Hellobdella sp. (Hirudinea); Insecta (Chironomus sp., Ephemeroptera, Trichoptera); Hyalella sp. (Amphipoda) y Physa venustula (Gastropoda); Sphaerium sp. (Bivalvia); tolerantes al enriquecimiento orgánico y concentraciones bajas de oxígeno, a excepción de los Amphipoda y los insectos con larvas tubicolas.

El número de individuos por laguna varió entre 7 y 35 , y se observó las mayores abundancias en las lagunas Tranca grande, Cucancocha y Ayhuin al igual que el número de especies (7 a 8) y la diversidad $\left(\mathrm{H}^{\prime}\right)(1,22$ a 1,26) (Tabla 3$)$.

El análisis de agrupamiento de las lagunas acuicolas (Fig. 2) permite diferenciar dos grupos, de condiciones de mesotrofia y eutrofia. El primer grupo estuvo formado por las lagunas Tranca Grande, Cucancocha y Ayhuin, las cuales se encuentran en 
Tabla 2. Valores promedios (febrero, mayo, agosto y octubre) para diferentes parámetros limnológicos en las lagunas altoandinas con cultivo intensivo de trucha, Junín, Perú, entre el año 1996 y el 2007.

\begin{tabular}{|c|c|c|c|c|c|c|c|}
\hline & \multicolumn{7}{|c|}{ Fósforo total $(\mu \mathrm{g} / \mathrm{L})$} \\
\hline & 1996 & 2002 & 2003 & 2004 & 2005 & 2006 & 2007 \\
\hline Tranca Grande & 6 & 30 & 30 & 28 & 28 & 25 & 25 \\
\hline Habascocha & 26 & 32 & 88 & 98 & 110 & 128 & 140 \\
\hline Pomacocha & 12 & 32 & 86 & 98 & 110 & 126 & 136 \\
\hline Huascacocha & 12 & 30 & 86 & 96 & 108 & 110 & 120 \\
\hline Tipicocha & 26 & 140 & 143 & 143 & 148 & 152 & 152 \\
\hline Cucancocha & 1 & 10 & 12 & 18 & 25 & 28 & 28 \\
\hline \multirow[t]{3}{*}{ Ayhuin } & 1 & 10 & 14 & 20 & 28 & 28 & 28 \\
\hline & \multicolumn{7}{|c|}{ Oxígeno disuelto (mg/L) } \\
\hline & 1996 & 2002 & 2003 & 2004 & 2005 & 2006 & 2007 \\
\hline Tranca Grande & 4,8 & 4,5 & 4,3 & 4,0 & 2,1 & 0,9 & 0,9 \\
\hline Habascocha & 7,0 & 1,2 & 1,0 & 1,0 & 0,9 & 0,7 & 0,7 \\
\hline Pomacocha & 8,0 & 1,8 & 1,0 & 1,0 & 0,9 & 0,7 & 0,8 \\
\hline Huascacocha & 4,8 & 2,0 & 1,0 & 1,0 & 0,9 & 0,7 & 0,7 \\
\hline Tipicocha & 3,0 & 2,0 & 1,0 & 1,0 & 0,9 & 0,7 & 0,9 \\
\hline Cucancocha & 6,9 & 4,9 & 4,3 & 4,0 & 2,1 & 2,1 & 1,6 \\
\hline \multirow[t]{3}{*}{ Ayhuin } & 6,9 & 4,9 & 4,3 & 4,0 & 2,1 & 2,1 & 1,6 \\
\hline & \multicolumn{7}{|c|}{ Transparencia (m) } \\
\hline & 1996 & 2002 & 2003 & 2004 & 2005 & 2006 & 2007 \\
\hline Tranca Grande & 7,4 & 5,6 & 4,7 & 4,6 & 4,8 & 4,4 & 4,4 \\
\hline Habascocha & 3,7 & 0,6 & 0,5 & 0,5 & 0,5 & 0,4 & 0,4 \\
\hline Pomacocha & 4,1 & 1,1 & 0,5 & 0,5 & 0,5 & 0,4 & 0,4 \\
\hline Huascacocha & 3,7 & 0,6 & 0,5 & 0,5 & 0,5 & 0,4 & 0,4 \\
\hline Tipicocha & 3,7 & 0,6 & 0,5 & 0,5 & 0,5 & 0,4 & 0,4 \\
\hline Cucancocha & 7,0 & 5,5 & 4,8 & 4,6 & 4,8 & 4,2 & 3,8 \\
\hline \multirow[t]{3}{*}{ Ayhuin } & 6,8 & 5,8 & 5,0 & 4,6 & 4,8 & 4,0 & 3,4 \\
\hline & \multicolumn{7}{|c|}{ Tamaño de grano $(\mu \mathrm{m})$} \\
\hline & 1996 & 2002 & 2003 & 2004 & 2005 & 2006 & 2007 \\
\hline Tranca Grande & 960 & 100 & 90 & 80 & 76 & $<62$ & $<62$ \\
\hline Habascocha & 600 & 100 & 90 & 80 & 76 & $<62$ & $<62$ \\
\hline Pomacocha & 650 & 100 & 90 & 80 & 76 & $<62$ & $<62$ \\
\hline Huascacocha & 550 & 100 & 90 & 80 & 76 & $<62$ & $<62$ \\
\hline Tipicocha & 300 & 100 & 90 & 80 & 76 & $<62$ & $<62$ \\
\hline Cucancocha & - & 1050 & 900 & 560 & 110 & $<62$ & $<62$ \\
\hline Ayhuin & - & 1050 & 900 & 450 & 120 & $<62$ & $<62$ \\
\hline
\end{tabular}

Tabla 6. Características del macrobentos en las lagunas altoandinas con cultivo intensivo de trucha, Junín, Perú, año 2007. Índice de riqueza de especies de Margalef, Indice de diversidad de Shannon $\left(H^{\prime}\right)$, índice de dominancia de Berger-Parker y equitabilidad (J') de Pielou.

\begin{tabular}{|c|c|c|c|c|c|c|}
\hline Lagunas & 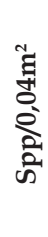 & 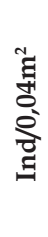 & 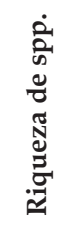 & 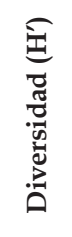 & 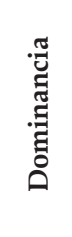 & 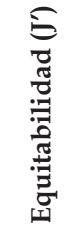 \\
\hline Tranca Grande & 7 & 35 & 1,42 & 1,22 & 0,94 & 0,44 \\
\hline Habascocha & 2 & 7 & 0,22 & 0,00 & 0,62 & 0,00 \\
\hline Tipicocha & 2 & 15 & 0,12 & 0,01 & 0,37 & 0,43 \\
\hline Huascacocha & 2 & 20 & 0,00 & 0,2 & 0,36 & 0,00 \\
\hline Pomacocha & 1 & 10 & 0,14 & 0,00 & 0,40 & 0,20 \\
\hline Cucancocha & 8 & 28 & 1,52 & 1,24 & 0,82 & 0,48 \\
\hline Ayhuin & 8 & 32 & 1,84 & 1,26 & 0,92 & 0,52 \\
\hline
\end{tabular}

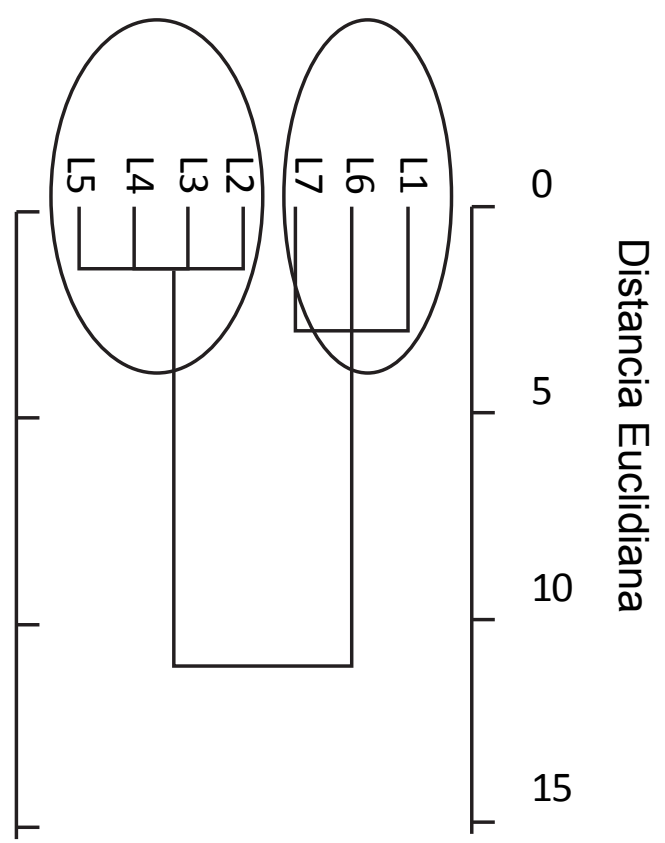

Figura 5. Dendrograma correspondiente al análisis de agrupamiento, de las lagunas altoandinas, Junín, Perú. Realizado con una matriz de densidades de macrobentos, distancia Euclideana y UPGMA. (1) Tranca grande, (2) Habascocha, (3) Tipicocha, (4) Huascacocha, (5) Pomacocha, (6) Cucancocha y (7) Ayhuin.

condiciones de mesotrofia y con profundidades mayores de 15 m. El segundo grupo estuvo formado las Lagunas Habascocha, Tipicocha, Huascacocha y Pomacocha, las cuales se encuentran en condiciones de eutrofia y profundidades menores de $15 \mathrm{~m}$.

Un problema importante en lagos y lagunas de alta montaña tropical, es el reciclado de la materia orgánica en los sedimentos (Dejoux \& Iltis 1991). En lagos andinos, con temperatura media de $11^{\circ} \mathrm{C}$, el reciclaje se procesa de manera mucha más lenta que en otros lagos tropicales. Callisto \& Esteves (1996) y Claude \& Oporto (2000) mencionan que en los lagos tropicales existe una rápida descomposición de la materia orgánica por las altas temperaturas de sus aguas $\left(>20^{\circ} \mathrm{C}\right)$, favorecidas por la turbulencia, sus cubetas pocas profundas, además, que la mayor parte de la materia orgánica es degradada en la columna de agua antes de alcanzar el sedimento.

La piscicultura intensiva puede tener un impacto negativo sobre el ecosistema lagunar, entre otras cosas por la acumulación de materia orgánica sobre los fondos, procedente de las excretas, materia orgánica muerta y la fracción de alimento no consumido (Buschmann, 2001). El aumento de materia orgánica en los fondos produce hipoxia y anoxia que conlleva a la disminución de la diversidad de las especies bentónicas y la predominancia de otras más tolerantes (Cornell \& Whoriskey 1993).

En el caso del cultivo de salmones, alrededor de un 75\% del nitrógeno, fósforo y carbono que ingresa al sistema como alimento, se pierde como alimento no ingerido, fecas y otros productos de excreción. Solo un $25 \%$ se convierte en carne para comercio. El fósforo se acumula principalmente en los sedimentos que se encuentran bajo las jaulas de cultivo, por lo que resulta un buen indicador de contaminación (Buschmann $\&$ Fortt 2005).

El tamańo de grano menor de $62 \mu \mathrm{m}$ del sedimento, encontrado en el presente estudio, guarda relación con el tamaño 
fino del alimento balanceado tipo peletizado. Tacon \& Cruz (1998), Kiang (1999) y Buschmann (2001) reportan la acumulacion en los sedimentos bajo jaulas de cultivo intensivo, la presencia de los alimentos balanceados peletizados, atribuyendo sus caracteristicas de rápido hundimiento, poca compactación y menor digestibilidad, representando un riesgo importante en el desarrollo de la acuicultura.

\section{Agradecimientos}

El presente trabajo fue financiado por el Vicerrectorado de Investigación de la UNMSM (proyectos CSI 961001201, 021001201, 031001045, 051001121, 061001041, 071001081). Igualmente nuestro agradecimiento a los Biólogos: Víctor Manuel Raez Oyola, Manuel Bedriñana Sosa, Milagros Ponce, Martín Silvera Solís, Andrés Mendoza, de la Dirección Regional de Producción, Junín, por la asistencia en campo. Al Dr. Enrique Vinatea Jaramillo por su invalorable colaboración en los trabajos de campo. Agradecemos a la Dirección Regional de Producción de Junín, por el préstamo de la embarcación y del transporte para los trabajos de campo.

\section{Literatura citada}

APHA, AWWA, WPCF. 1993. Métodos Normalizados para el análisis de agua potables y residuales. 17a ed. Ediciones Díaz de santos S.A. 1-1 a 10-220 pp

Buschmann A.H. 2001. Impacto ambiental de la acuicultura. El estado de la investigación en Chile y el Mundo. Registro de Problemas Públicos $\mathrm{N}^{\circ}$ 4. Terram Publicaciones. 67 pp.

Buschmann A.H. \& A. Fortt. 2005. Efectos ambientales de la acuicultura intensiva y alternativas para un desarrollo sustentable Revista Ambiente y Desarrollo 21(3): 58-64.

Callisto M. \& F. A. Esteves. 1996. Composição granulométrica do sedimento de um lago amazônico impactado por rejeito de bauxita e um lago natural. Acta Limnologica Brasiliensia 8: $115-126$.

Carrit D. \& J. Carpenter. 1966. Comparison and evaluation of currently employed modifications of the Winkler method for determining dissolved oxygen in sea water. J. Mar. Res., 24:286-318.
Claude M. \& J. Oporto. 2000. Salmonicultura en Chile: Aspectos Sociales, economicos y ambientales. Colección Sin norte. Lom Ediciones. 72 pp

Cornell G.E. \& F.G.Whoriskey. 1993. The effects of rainbow trout (Oncorhynchus mykiss) cage culture on the water quality, zooplankton, benthos, benthos and sediments of Lac du Passage, Quebec. Aquaculture 109: 101-117.

Dean W.C. 1974. Determination of carbonate and organic matter in calcareous sediments by on ignition: comparision with other methods. Jour. Sed. Petrology 44: 242-248.

Dejoux C. \& A. Iltis. 1991. El Lago Titicaca. Síntesis del Conocimiento Limnológico. Actual ORSTM Imp. Talleres Gráficos Hisbol. La Paz- Bolivia. 578 pp.

Hansen P.V., A.D. Ervick, F.H.Schaanming, et al. 2001. Regulating the local environmental impact of intensive marine fish farming. The concept of the Mom System (modeling Ongrowing fish form-Monitoring). Acuaculture 194:75-92.

Huaman M.P., M.G. Mariano, M.E. Chanco, et al. 2002. Estructura del macrobentos de la laguna de Paca, Junín. Rev peru biol. 9(1):29-38.

Kiang J.K. 1999. The principles of extruding fishfeeds, Feed Tech 3(6): 48-49.

Krumbein W.C. \& F.J. Pettijohn. 1938. Manual of sedimentary petrography. 91-181.

Magurran A.E. 1988. Ecological diversity and its measurement, Taylor \& Francis. 198 pp.

Ministerio de Pesquería. 1997. Pesca y Medio Ambiente en el Titicaca. Manual de Capacitación. Proyecto Padespa. Edic. MMD.

Phillips M.J., M.C.M. Beveridge \& L.G. Ross. 1985. The environmental impact of salmonid cage culture on inland fisheries: present status and future trends. Journal of Fish Biology 27(sa): 123-137.

SEDAPAL. 1999. El País de las lagunas. Historia y Ecología de la Puna de Junín. Sedapal. Taller Gráfica Biblios. Lima, Perú.

Tacon, A., E. Cruz. 1999. Gestión de la Acuicultura "Alimentación y Nutrición". En: Acuicultura Sostenible: Desarrollo y Comercio. Lima, Perú, 9-11 Junio, 1999. 36pp. 